\title{
Development of an Unnatural Amino Acid Incorporation System in the Actinobacterial Natural Product Producer Streptomyces venezuelae ATCC 15439
}

\author{
Jingxuan $\mathrm{He}^{\dagger}$, Briana Van Treeck ${ }^{\dagger}$, Han B. Nguyen ${ }^{\dagger}$, and Charles E. Melançon $\mathrm{III}^{\star}, \dagger, \ddagger, \S$ \\ tDepartment of Chemistry and Chemical Biology, University of New Mexico, Albuquerque, NM \\ 87131-0001, United States \\ ¥Department of Biology, University of New Mexico, Albuquerque, NM 87131-0001, United States \\ $\S$ Center for Biomedical Engineering, University of New Mexico, Albuquerque, NM 87131-0001, \\ United States
}

\begin{abstract}
Many Actinobacteria, most notably Streptomyces, produce structurally diverse bioactive natural products, including ribosomally synthesized peptides, by multistep enzymatic pathways. The use of site-specific genetic incorporation of unnatural amino acids to investigate and manipulate the functions of natural product biosynthetic enzymes, enzyme complexes, and ribosomally-derived peptides in these organisms would have important implications for drug discovery and development efforts. Here, we have designed, constructed, and optimized unnatural amino acid systems capable of incorporating p-iodo-L-phenylalanine and p-azido-L-phenylalanine sitespecifically into proteins in the model natural product producer Streptomyces venezuelae ATCC 15439. We observed notable differences in the fidelity and efficiency of these systems between $S$. venezuelae and previously used hosts. Our findings serve as a foundation for using an expanded genetic code in Streptomyces to address questions related to natural product biosynthesis and mechanism of action that are relevant to drug discovery and development.
\end{abstract}

\section{Keywords}

unnatural amino acids; Streptomyces; p-azido-ı-phenylalanine; p-iodo-ı-phenylalanine; green fluorescent protein; natural products

\begin{abstract}
High G/C Gram-positive Actinobacteria, most notably members of genus Streptomyces, are known for their ability to produce a variety of structurally complex bioactive natural products, including polyketides, non-ribosomal and ribosomal peptides, terpenoids, and other classes of molecules. Natural products are biosynthesized using a combination of primary metabolites and specialized secondary metabolite precursors by tightly regulated ${ }^{1}$ and highly coordinated ${ }^{2}$ multistep enzymatic pathways. The enzymes in these pathways
\end{abstract}

*cemelanc@unm.edu.

Notes

The authors declare no competing financial interests. 
often rely on specific protein-protein interactions between domains and/or discrete polypeptides for biosynthesis to proceed correctly. ${ }^{2,3}$ Codon usage and genetic regulation in Actinobacteria also differ significantly from more commonly used bacterial hosts such as $E$. coli. ${ }^{4,5}$ Because of the unique metabolic, genetic, and biochemical features of actinobacterial natural product biosynthetic pathways, functional heterologous expression of these pathways in E. coli is extremely challenging. ${ }^{6,7}$ As a result, genetically tractable Streptomyces strains are often the preferred hosts for heterologous expression of Actinobacterial natural product biosynthetic enzymes and pathways. $4,5,8$

Several genetically manipulable Streptomyces strains, including Streptomyces coelicolor A3(2) and its mutants ${ }^{9}$ Streptomyces lividans strains, Streptomyces albus J1074, Streptomyces venezuelae ATCC $15439^{10}$ and Streptomyces avermitilis SUKA mutants ${ }^{11}$ are commonly used as hosts for expressing a variety of natural product enzymes and pathways from diverse Actinobacteria. ${ }^{4,5,8}$ However many of the genetic, chemical biology, and synthetic biology tools available for use in E. coli have yet to be developed for these model Streptomyces strains. Among these tools, site-specific genetic incorporation of unnatural amino acids (UAAs) using evolved orthogonal aminoacyl-tRNA synthetase/amber suppressor tRNA (aaRS/tRNAcuA) pairs is one of the most versatile and powerful for studying and manipulating protein function in living cells ${ }^{12,13}$ (Figure la). Systems for incorporating nearly 100 different UAAs for various applications have been developed for a variety of hosts, including E. coli, S. cerevisiae, $P$. pastoris, $M$ smegmatis, mammalian cells, C. elegans, and D. melanogaster. ${ }^{13}$ InE. coli, variants of the $M$ jannaschii tyrosyl-tRNA synthetase/amber suppressor tRNA (MjTyrRS/MjtRNATyrcuA) pair ${ }^{12,13}$ evolved to incorporate specific UAAs are the most widely used aaRS/tRNAcuA pairs. Of the UAAs incorporated by evolved variants of MjTyrRS, the photocrosslinker p-benzoyl-Lphenylalanine ${ }^{14}$ (pBpa), the photocrosslinking and Huisgen cycloaddition partner p-azido-Lphenylalanine ${ }^{15}$ (pAzPhe), and the cross coupling amino acidp-iodo-s-phenylalanine ${ }^{16,17}$ (pIPhe) (Figure lb) are among the most useful. pAzPhe and pBpa have been used extensively to identify interacting proteins and to map protein-protein interactions. ${ }^{12,13}$ pAzPhe and pIPhe have been used to chemically couple various functionalities, including chemical tags and fluorophores, to proteins via biorthogonal reactions. ${ }^{12,13,17}$

In an effort to extend the capabilities afforded by UAA incorporation to study and manipulate natural product biosynthetic enzymes and pathways and ribosomally synthesized peptides, including those from the well-studied lantibiotic subclass, in Actinobacteria, we have developed and optimized an UAA incorporation system for the model streptomycete $S$. venezuelae ATCC 15439. The system relies on expression of a synthetic operon containing UAA-specific variants of MjTyrRS, MjtRNAryrcuA. and an amber suppressible green fluorescent protein (GFP-TAG) reporter, refactored to function properly in S. venezuelae. We demonstrate that amber suppression of GFP-TAG occurs in S. venezuelae only when MjTyrRS and MjtRNATyrcuA are co-expressed; and that incorporation of pIPhe and pAzPhe into GFP can be achieved with good to excellent fidelity under optimized conditions. We observed notable differences in the behavior of UAA incorporation systems between $S$. venezuelae and previously employed hosts, including an inability of the $S$. venezuelae translational machinery to efficiently accommodate $\mathrm{pBpa}$ charged 
MjtRNAryrcuA; and a decreased selectivity of engineered MjTyrRS variants for their cognate UAAs over endogenous proteinogenic amino acids. We identified tyrosine and to a lesser extent phenylalanine as endogenous amino acids toward which MjTyrRS variants retain detectable catalytic activity in S. venezuelae. These results demonstrate the feasibility of UAA incorporation in Streptomyces and identify specific features of the system as targets for further optimization. The system developed here provides a platform for interrogating protein-protein interactions in natural product biosynthetic enzymes and multi-enzyme complexes via UAA-mediated photocrosslinking; and for incorporating bio-orthogonal chemical moieties into ribosomally synthesized peptides, in a cellular context conducive to the proper functioning of natural product biosynthetic enzyme systems.

We chose $S$. venezuelae ATCC 15439, best known for its production of the methymycin/ pikromycin family of macrolide antibiotics ${ }^{18}$ and for its use in engineered production of macrolide derivatives bearing altered sugar moieties ${ }^{19}$ as the host for developing the UAA incorporation system. S. venezuelae ATCC 15439 is among the most rapidly growing model Streptomyces hosts (doubling time of $-1 \mathrm{~h}^{10}$ ), grows in a dispersed manner in liquid culture, and can be genetically manipulated easily using either conjugation or protoplast transformation for introduction of plasmid DNA, making it a convenient host for synthetic biology applications. We used the E. coli-Streptomyces shuttle vector, $\mathrm{pCMld}^{20}$ bearing $\mathrm{SCP} 2 *$ and ColElorigins of replication and an apramycin resistance marker, for construction of all plasmids expressing functional elements of the UAA incorporation system.

First, we constructed a series of four vectors (Figure 2a) to individually test proper functioning of MjTyrRS, MjtRNATyrcu\& and the GFP reporter in S. venezuelae. Plasmid pSUA5, which contains the wild-type humanized enhanced GFP (eGFP) reporter gene with C-terminal histidine tag (eGFP-His) under control of constitutive Streptomyces promoter $\mathrm{SF} 14^{21}$ was designed to test expression of the reporter gene. Plasmid pSUA4, which contains eGFP-His with an amber stop codon at position 150 (eGFP-150TAG), was designed to test whether $S$. venezuelae has endogenous amber suppression capability, which would be undesirable. Plasmid pSUA3, which contains eGFP-150TAG and an optimized MjtRNA1)TCUA Nap $1^{22}$ with Streptomyces coelicolor tRNA promoter and flanking sequences (see Supporting Information for details), was designed to test MjtRNATyrcuA orthogonality to endogenous aaRS in S. venezuelae, which is critical for system functionality. Plasmid pSUA2, which contains eGFP-150TAG, MjtRNA1)TcuA. and Cterminally histidine tagged MjTyrRS (MjTyrRS-His) under control of the SF14 promoter, was designed to test functionality of the amber suppression system as a whole.

To examine the performance of these constructs, we individually transferred pSUA2, pSUA3, pSUA4, and pSUA5 to $S$. venezuelae by protoplast transformation and carried out nickel-NTA affinity purification of eGFP (or the mixture of MjTyrRS and eGFP in the case of pSUA2) from each mutant and anti-His Western blotting. The results (Figure 2b, lanes 2-4) demonstrate that $S$. venezuelae harboring pSUA5 expresses eGFP (Figure 2b, lane 4); and that $S$. venezuelae harboring pSUA4 does not produce detectable levels of eGFP (Figure $2 \mathrm{~b}$, lane 3), indicating that no endogenous amber suppression system is present in $S$. venezue/ae. However, neither amber suppressed eGFP nor MjTyrRS could be detected in $S$. venezuelae harboring pSUA2 (data not shown). Examination of the MjTyrRS sequence 
revealed a large number of codons that occur infrequently in Streptomyces genes, including 18 TTA codons, which is the least used codon in Streptomyces. ${ }^{23}$ Reasoning that the lack of expression of MjTyrRS and the inability to produce amber suppressed eGFP were likely due to the presence of rare codons in MjTyrRS, we constructed a pSUA2 variant, opt-pSUA2, containing a codon optimized version of MjTyrRS-His (MjTyrRS-opt) and tested its functionality in S. venezue/ae by affinity purification of the mixture of MjTyrRS-opt and eGFP, and anti-His Western blotting. The results (Figure 2, lane 1) indicate that MjTyrRSopt is expressed at a high level in S. venezuelae, but more importantly, that amber suppressed eGFP is produced, and thus that the amber suppression system as a whole is functional in S. venezuelae. In light of this result, the fact that $S$. venezuelae harboring pSUA3 (Figure 2, lane 2) does not produce detectable levels of eGFP also indicates that MjtRNATyrcuA is orthogonal in S. venezuelae. Removal of the C-terminal histidine tag from MjTyrRS-opt in opt-pSUA2 followed by expression in S. venezuelae, affinity purification, and anti-His Western blotting further confirmed expression of amber suppressed eGFP (Figure 2, lane 5).

Next, we constructed three MjTyrRS-opt variants with the appropriate mutations to confer specificity for the UAAs pBpa, pAzPhe, and pIPhe ${ }^{14-16}$ (p-benzoyl-L-phenylalaninyl-, $p$ azido-ı-phenylalaninyl-, and p-iodo--_-phenylalaninyl-tRNA synthetases; pBpaRS, pAzPheRS, pIPheRS, respectively) and cloned them into the pSUA2 vector inplace ofMjTyrRS, resulting inpSUAl-pBpaRS, pSUAl-pAzPheRS, and pSUAl-plPheRS (Figure $3 a)$. We also replaced the eGFP-150TAG gene in each of these three pSUAl variants and in control vector pSUA2 with a codon optimized, C-terminally histidine tagged version of the improved GFP variant superfolder GFP (sfGFP) ${ }^{24}$ with an amber stop codon in place of the tyrosine codon at position 151 (sfGFP-151TAG); and replaced eGFP in control vector pSUA5 with an sfGFP variant lacking an amber stop codon (Figure 3a). S. venezuelae harboring these constructs were used for subsequent studies. The ability of $S$. venezuelae harboring pSUAl-pBpaRS, pSUAl-pAzPheRS, and pSUAl-pIPheRS to produce UAAcontaining GFP variants was first tested by affinity purification and anti-His Western blotting of GFP produced in the presence and absence of $1 \mathrm{mM}$ of each cognate UAA. Western blotting results ofGFP obtained from the three constructs grown in the presence of cognate UAAs (Figure 3b, lanes 1, 3, and 5) suggest that pIPhe and pAzPhe, but not pBpa, are incorporated into GFP. However, in contrast to previous :findings in E. coli ${ }^{14-16}$ analysis of -, these three mutants grown in the absence of cognate UAAs (Figure 3b, lanes 2, 4, and 6) indicates that endogenous amino acids are recognized to varying extents by pBpaRS, pAzPheRS, and pIPheRS in S. venezuelae, with pIPheRS showing only trace activity toward endogenous amino acids, pBpaRS showing moderate activity, and pAzPheRS showing high activity. These results suggest that undesirable background incorporation of endogenous amino acids may occur in the presence of cognate UAAs. Also, the surprising finding that GFP was not produced in $S$. venezuelae harboring pSUAl-pBpaRS grown in the presence of $1 \mathrm{mM} \mathrm{pBpa} \mathrm{(Figure} 3 \mathrm{~b}$, lane 1), but was produced by the same strain grown without $\mathrm{pBpa}$ (Figure 3b, lane 2) suggests that pBpa-charged MjtRNATyrcuA is produced intracellularly, but that it is unable to be accommodated by the $S$. venezuelae translational machinery to a sufficient extent to allow detection of GFP by Western blot. We also quantified the relative expressed yields of GFP proteins obtained after small scale purification by measuring 
fluorescence, and found that amber suppression efficiencies range from 7-12\% as compared to wild-type GFP (Figure 3c).

To confirm incorporation of pIPhe and pAzPhe into GFP in S. venezuelae mutants harboring pSUAl-pIPheRS and pSUAl-pAzPheRS we carried out large-scale expression (1.6 L culture), purification, and intact protein electrospray ionization mass spectrometry (ESI-MS) of GFP proteins produced by each mutant grown in the presence of $1 \mathrm{mM}$ cognate UAA (Figure 4a, lanes 1 and 3). GFP was also purified from a $1.6 \mathrm{~L}$ culture of $S$. venezuelae harboring pSUA2 (Figure 4a, lane 4) and analyzed by ESI-MS to verify homogeneous incorporation of tyrosine. Expressed. yields were $0.19,0.49$, and $0.46 \mathrm{mg} ! \mathrm{L}$ for $S$. venezuelae harboring pSUAl-pIPheRS, pSUAl-pAzPheRS, and pSUA2 respectively; and isolated. protein yields from these mutants were 22,22 , and $45 \mu \mathrm{g}$, respectively (see Supporting Information for protein quantification). Mass spectrometry results indicated that homogeneous tyrosine-containing protein and homogeneous pIPhe-containing protein were produced by $S$. venezuelae harboring pSUA2 and pSUAl-pIPheRS, respectively (Figure 5a, b; Figure S2, S3). However, we observed only 33\% incorporation of pAzPhe from $S$. venezuelae harboring pSUAl-pAzPheRS, with the remaining protein having a mass consistent with incorporation of either tyrosine or p-amino---phenylalanine (a known intermediate in the biosynthesis of the Streptomyces natural product chloramphenicol ${ }^{25}$, and possible product of enzymatic reduction of $\mathrm{pAzPhe}{ }^{26}$ ), which differ in mass by only one dalton (Figure 5c, Figure S4). Therefore, pAzPheRS shows poor selectivity for pAzPhe in $S$. venezuelae. We further confirmed the genetic incorporation of pAzPhe by incubating GFP purified from $S$. venezuelae harboring pSUAl-pAzPheRS grown either in the presence or the absence of $1 \mathrm{mM}$ pAzPhe with the strained alkyne dibenzocyclooctyne-PEG 4 -biotin (DBCO-biotin), and carrying out streptavidin Western blotting. The results show that GFP produced in the presence of pAzPhe, but not that produced in the absence of pAzPhe, is labeled by DBCO-biotin (Figure 4b).

To attempt to improve the fidelity of pAzPhe incorporation, we tested several parameters: pAzPheRS sequence, media composition, and UAA concentration. First, reasoning that other MjTyrRS variants evolved in E. coli to recognize pAzPhe may have improved selectivity for pAzPhe over endogenous amino acids, we constructed new pSUAl derivatives in which pAzPheRS was replaced by each of three previously reported, alternative pAzPheRS sequences (pAzPheRS-2, pAzPheRS-4, and pAzPheRS-5). ${ }^{15} \mathrm{We}$ first tested the performance of these constructs by small scale expression and purification followed by fluorescence quantification and Western blotting. Two of these (pAzPheRS-2, pAzPheRS-4) showed differential amounts of protein in the presence versus the absence of pAzPhe, whereas one (pAzPheRS-5) did not (Figure Sl). However large-scale expression (1.6 L), purification, and ESI-MS analysis of GFP from S. venezuelae harboring pSUAlpAzPheRS-2 and pSUAl-pAzPheRS-4 showed that neither variant had improved pAzPhe incorporation fidelity (32\% for pAzPheRS-2 and $27 \%$ for pAzPheRS-4) with the remaining protein having mass consistent with incorporation of tyrosine or $p$ amino-ı-phenylalanine (Figure 5d, e; Figure S5, S6). Next, large-scale growth (1.6 L) in Streptomyces minimal media (MM) ${ }^{27}$,which contains no amino acids, was tested to attempt to reduce the intracellular concentration of endogenous amino acids that compete with pAzPhe. However, 
GFP isolated from S. venezuelae harboring pSUAl-pAzPheRS grown in MM supplemented with $1 \mathrm{mM}$ pAzPhe showed reduced pAzPhe incorporation fidelity (14\%) (Figure Sf, Figure S7). Finally, we attempted to out compete endogenous amino acids by large-scale growth (1.6 L) of $S$. venezuelae harboring pSUAl pAzPheRS in the presence of S mM pAzPhe. The expressed yield of GFP was $0.24 \mathrm{mg} / \mathrm{L}$. The resulting purified protein $(30 \mu \mathrm{g}$, Figure $4 \mathrm{a}$, lane 2) contained $77 \%$ pAzPhe, with the remaining $23 \%$ having mass consistent with incorporation of tyrosine or p-amino-L-phenylalanine (Figure Sg; Figure SS). Proteins with this level ofpAzPhe incorporation are suitable for many downstream applications, including in vitro and in vivo photocrosslinking experiments to investigate protein-protein interactions. Thus, we have developed and optimized engineered $S$. venezuelae hosts capable of sitespecific incorporation of plPhe and pAzPhe into target proteins.

Because of the observed incorporation of endogenous ammo acids in S. venezuelae when pBpaRS, pAzPheRS, and pIPheRS were employed in the absence of cognate UAA (Figure $3 \mathrm{a}$, lanes 2,4 , and 6 , respectively); and the observed incorporation of non-cognate amino acid when pAzPheRS, pAzPheRS-2, and pAzPheRS-4 were employed in the presence ofpAzPhe (Figure Sc-g), neither of which occurred in the E. coli host in which these aaRS were originally developed, we carried out additional experiments to identify the amino acid(s) being incorporated. The results of these experiments are important for future optimization and expansion of UAA incorporation technology in S. venezuelae through development of engineered aaRS with improved selectivity for cognate UAAs and metabolic engineering to reduce or eliminate endogenous production of amino acids that compete with cognate UAAs as substrates for aaRS.

First, we carried out large-scale expression, purification, and ESI-MS of GFP from $S$. venezuelae harboring pSUAl-pIPheRS, pSUAl-pAzPheRS, and pSUAl-pBpaRS grown in the absence ofUAA. The results showed that a mixture of phenylalanine (71\%) and either tyrosine or p-amino-L-phenylalanine (29\%) was incorporated when plPheRS was employed (Figure Sh, Figure S9); a mixture of phenylalanine (42\%) and either tyrosine or p-amino-Lphenylalanine (S8\%) was incorporated when pAzPheRS was employed (Figure 5i, Figure $\mathrm{SlO}$ ); and homogeneous tyrosine or p-amino-L-phenylalanine was incorporated when pBpaRS was employed (Figure 5j, Figure Sll). Thus, in the absence of UAA. each aaRS is capable of charging MjtRNATyrcuA with endogenous amino acid(s); and each aaRS displays a distinct degree of catalytic competence for each of these amino acids.

Next, we carried out experiments to distinguish between incorporation of tyrosine andpamino-ı-phenylalanine by engineered aaR.S in S. venezuelae. We first searched the recently sequenced S. venezuelae ATCC 15439 genome (manuscript in preparation) for homologues of known p-amino---phenylalanine biosynthetic genes PapA, PapB, and PapC. ${ }^{25}$ No such homologues were identified, suggesting that $S$. venezuelae ATCC 15439 is unable to biosynthesize p-amino-L-phenylalanine and therefore that tyrosine is incorporated by engineered aaR.S. To further test this notion, we generated an sfGFP variant in which the codon for tyrosine 66, which forms part of the GFP fluorophore, was replaced by an amber stop codon (sfGFP-Y66TAG). Replacement of Y66 by various UAAs, includingp-amino-Lphenylalanine, has been shown to result in specific shifts in excitation and absorption maxima of the GFP fluorophore.28- ${ }^{30}$ Among the various combinations of aaRS and 
expression conditions we tested that resulted in detectable levels of non-cognate amino acid incorporation (Figure 5c-j), only pBpaR.S employed in the absence of UAA resulted in a mass spectral peak consistent with homogeneous incorporation of either tyrosine orp-aminoL-phenylalanine. We therefore constructed a pSUAl-pBpaR.S derivative containing sfGFPY66TAG (pSUA1-pBpaRS-Y66TAG) and isolated GFP from S. venezuelae harboring this construct grown in the absence of UAA. The mass spectrum of the purified protein is essentially identical to that of GFP isolated from $S$. venezuelae harboring pSUAl-pBpaR.S grown in the absence of UAA (Figure 5k, Figure S12). We compared the fluorescence excitation and emission spectra of the resulting GFP with those of GFP isolated from $S$. venezuelae harboring pSUA2, which possesses tyrosine encoded by a sense codon at position 66. The two were found to be indistinguishable, with excitation and emission maxima of 485 and $510 \mathrm{~nm}$, respectively, which are indicative of a tyrosine derived fluorophore (Figure 6). sfGFP containing p-amino-ı-phenylalanine at position 66 has been reported to possess excitation and emission maxima of 446 and 498 run, respectively. ${ }^{30}$ Therefore, the fluorescence spectra of GFP isolated from $S$. venezuelae harboring pSUA1pBpaRS-Y66TAG are consistent with incmporation of tyrosine, and not p-amino-Lphenylalanine, by pBpaRS. However, due to the decreased brightness of sfUFP with pamino-L-phenylalanine at position 66 (11\% of GFP with tyrosine at this position) ${ }^{30}$ we cannot exclude the possibility of incorporation of a low level ofp-amino-L-phenylalanine. Also, since this experiment was conducted using pBpaRS, we cannot rule out incorporation ofp-amino-s-phenylalanine by pAzPheRS or plPheRS. However, when taken together, the genomic, fluorescence, and mass spectral data suggest that tyrosine, and not p-amino-Lphenylalanine, is incorporated not only by $\mathrm{pBpaRS}$ in the absence of $\mathrm{pBpa}$, but also by pAzPheRS in the presence and absence of pAzPhe, and by pIPheRS in the absence of pIPhe. Thus, we plan to focus future $S$. venezuelae UAA incorporation system optimization efforts on decreasing the catalytic competence of engineered aaRS toward tyrosine.

In summary, we have designed, constructed, and optimized amber suppression-based UAA incorporation systems capable of installing pIPhe and pAzPhe site-specifically into proteins in Streptomyces venezuelae ATCC 15439 with good to excellent fidelity and with sufficient yield for most applications. Development of an UAA incorporation platform in Streptomyces extends the scope of this powerful approach to studying and manipulating the functions of actinobacterial proteins, protein complexes, and peptides in their native cellular contexts. This is especially important in light of the significant challenges associated with heterologous expression of functional multi-enzyme complexes involved in natural product biosynthesis in non-streptomycete hosts. Among the many possible applications, we envision using this technology to identify and map protein-protein interactions in natural product biosynthetic multi-enzyme complexes via UAA-mediated photocrosslinking; and to incorporate bio-orthogonal chemical moieties into ribosomally synthesized peptides to facilitate their detection, isolation, and studies of their mechanisms of action.

In the process of developing and optimizing the S. venezuelae VAA incorporation system, we observed two notable differences in system performance between $S$. venezuelae and previously employed hosts: an inability to incorporate a detectable level of pBpa into the target GFP protein, and a decreased selectivity of engineered MjTyrRS variants for their 
cognate UAAs over endogenous proteinogenic amino acids. We suggest that the former may be due to more stringent discrimination of the $S$. venezuelae ribosome and/or elongation factor $\mathrm{Tu}(\mathrm{EF}-\mathrm{Tu})$ than their E. coli homologues against tRNA charged with bulky UAAs such as pBpa. To investigate the latter, we carried out additional experiments to identify the endogenous amino acid(s) being incorporated. We demonstrated that plPheRS and pAzPheRS accept phenylalanine as a substrate, but only in the absence of their cognate UAAs; and obtained genomic, and fluorescence and mass spectral evidence for the incorporation of tyrosine, and not p-amino-L-phenylalanine, when pBpaRS is employed in the absence of pBpa. Based on the combined evidence, we suggest that tyrosine is incorporated with varying catalytic efficiencies in S. venezuelae by all engineered aaRS employed in this study in the absence of their cognate UAAs, and by all three pAzPheRS variants in the presence of $1 \mathrm{mM}$ pAzPhe. The varying abilities of the MjTyrRS variants employed in this study to utilize aromatic amino acids tyrosine and to a lesser extent phenylalanine as substrates are consistent with elevated intracellular concentrations of these amino acids in S. venezuelae relative to the $E$. coli host in which these aaRS were evolved . We suggest that pAzPheRS variants with sufficiently improved selectivity for pAzPhe to allow homogeneous pAzPhe incorporation in S. venezuelae at the standard $1 \mathrm{mM}$ concentration could be obtained by supplementing the media used in the negative selection step of aaRS directed evolution in E. coli, with tyrosine.

\section{Methods}

Experimental procedures are described in detail in the Supporting Information.

Due to the high expression of MjTyRS-opt relative to eGFP (lane 1), the signal from which interfered with clear visualization of the GFP band, the blot was initially imaged for $5 \mathrm{~s}$; and the upper portion of lane 1 containing MjTyrRS-opt was then excised from the blot (excised portion is marked with dashed lines), and the blot was then imaged for an additional $135 \mathrm{~s}$.

\section{Supplementary Material}

Refer to Web version on PubMed Central for supplementary material.

\section{Acknowledgements}

We gratefully acknowledge Kenneth D. Sherrell at the University of New Mexico for assistance with protein mass spectrometry; Peter G. Schultz at the Scripps Research Institute for providing plasmids harboring MjTyrRS, evolved MjTyrRS variants, and MjtRNATyrcuA; Hung-wen Liu at The University of Texas at Austin for providing S. venezuelae ATCC 15439, and Fu-sen Liang at the University of New Mexico for providing the plasmid harboring the eGFP gene. This work is supported by NIH NM-INBRE grant P20 GMl 03451 (C. E. M.) and by the University of New Mexico.

\section{References}

(1). Rokem JS, Lantz AE, Nielsen J. Systems biology of antibiotic production by microorganisms. Nat. Prod. Rep. 2007; 24:1262-1287. [PubMed: 18033579]

(2). Weissman KJ. The Structural biology of biosynthetic megaenzymes. Nat. Chem. Biol. 2015; 11:660-670. [PubMed: 26284673]

(3). Xu W, Qiao K, Tang Y. Structural analysis of protein-protein interactions in type I polyketide synthases. Crit. Rev. Biochem. Mol. Biol. 2013; 48:98-122. [PubMed: 23249187] 
(4). Ongley SE, Bian X, Neilan BA, Miiller R. Recent advances in the heterologous expression of microbial natural product biosynthetic pathways. Nat. Prod. Rep. 2013; 30:1121-1138. [PubMed: 23832108]

(5). Zhang H, Boghigian BA, Armando J, Pfeifer BA. Methods and options for heterologous production of complex natural products. Nat. Prod .Rep. 2011; 28:125-151. [PubMed: 21060956]

(6). Li J, Neubauer P. Escherichia coli as a factory for heterologous production of nonribosomal paptides and polyketides. New Biotechnol. 2014; 31:579-585.

(7). Yuzawa S, Kim W, Katz L, Keasling JD. Heterologous production ofpolyketides by modular type I polyketide synthases in Escherichia coli. Curr. Opin. Biotechnol. 2012; 23:727-735. [PubMed: 22244790]

(8). Baltz RH. Streptomyces and Saccharopolyspora hosts for heterologous expression of secondary metabolite gene clusters. J. Ind. Microbiol. Biotechnol. 2010; 37:759-772. [PubMed: 20467781]

(9). Gomez-Escribano JP, Bibb MJ. Heterologous expression of natural product biosynthetic gene clusters in Streptomyces coelicolor: from genome mining to manipulation of biosynthetic pathways. J. Ind. Microbiol. Biotechnol. 2014; 41:425-431. [PubMed: 24096958]

(10). Kim EJ, Yang I, Yoon YJ. Developing Streptomyces venezuelae as a cell factory for the production of small molecules used in drug discovery. Arch. Pharm. Res. 2015; 38:1606-1616. [PubMed: 26211662]

(11). Ikeda H, Shin-ya K, Omura S. Genome mining of the Streptomyces avermitilis genome and development of genome-minimized hosts for heterologous expression ofbiosynthetic gene clusters. J. Ind. Microbiol. Biotechnol. 2014; 41:233-250. [PubMed: 23990133]

(12). Liu CC, Schultz PG. Adding New Chemistries to the Genetic Code. Annu. Rev. Biochem. 2010; 79:413-444. [PubMed: 20307192]

(13). Chin JW. Expanding and Reprogramming the Genetic Code of Cells and Animals. Annu. Rev. Biochem. 2014; 83:379-408. [PubMed: 24555827]

(14). Chin JW, Martin AB, King DS, Wang L, Schultz PG. Addition of a photocrosslinking amino acid to the genetic code of Escherichia coli. Proc. Nat. Acad. Sci. USA. 2002; 99:11020-11024. [PubMed: 12154230]

(15). Chin JW, Santoro SW, Martin AB, King DS, Wang L, Schultz PG. Addition ofp-azido-Lphenylalanine to the genetic code of Escherichia coli. J. Am. Chem. Soc. 2002; 124:9026-9027. [PubMed: 12148987]

(16). Xie J, Wang L, Wu N, Brock A, Spraggon G, Schultz PG. The site-specific incorporation of piodo-L-phenylalanine into proteins for structure determination. Nat. Biotechnol. 2004; 22:12971301. [PubMed: 15378068]

(17). Yang M, Li J, Chen PR. Transition metal-mediated bioorthogonal protein chemistry in living cells. Chem. Soc. Rev. 2014; 43:6511-6526. [PubMed: 24867400]

(18). Xue Y, Zhao L, Liu H.-w. Shennan DH. A gene cluster for macrolide antibiotic biosynthesis in Streptomyces venezuelae: architecture of metabolic diversity. Proc. Nat. Acad. Sci. USA. 1998; 95:12111-12116. [PubMed: 9770448]

(19). Thibodeaux CJ, Melançon CE III, Liu H.-w. Natural-Product Sugar Biosynthesis and Enzymatic Glycodiversification. Ang. Chem. Int. Ed. 2008; 47:9814-9859.

(20). Melançon CE III, Liu H.-w. Engineered Biosynthesis of Macrolide Derivatives Bearing the NonNatural Deoxysugars 4-epi-d-Mycaminose and 3-N-Monomethylamino-3-Deoxy-d-Fucose. J. Am. Chem. Soc. 2007; 129:4896-4897. [PubMed: 17388593]

(21). Labes G, Bibb M, Wohlleben W. Isolation and characterization of a strong promoter element from the Streptomyces ghanaerisis phage 119 using the gentamicin resistance gene $(\mathrm{aacCl})$ of Tn1696 as reporter. Microbial. 1997; 143:1503-1512.

(22). Guo J, Melançon CE III, Lee HS, Groff D, Schultz PG. Evolution of Amber Suppressor tRNAs for Efficient Bacterial Production of Proteins Containing Nonnatural Amino Acids. Ang. Chem. Int. Ed. 2009; 48:1-5.

(23). Chater KF, Chandra G. The use of rare UUA codon to define "expression space" for genes involved in secondary metabolism, development, and environmental adaptation in Streptomyces, .! Microbiol. 2008; 46:1-11. 
(24). Pedelacq J-D, Cabantous S, Tran T, Terwilliger TC, Waldo GS. Engineering and characterization of a superfolder green fluorescent protein. Nat. Biotechnol. 2006; 24:79-88. [PubMed: 16369541]

(25). Yanai K, Sumida N, Okakura K, Moriya T, Watanabe M, Murakami T. Para-position derivatives of fungal anthelmintic cyclodepsipeptides engineered with Streptomyces venezuelae antibiotic biosynthetic genes. Nat. Biotechnol. 2004; 22:848-855. [PubMed: 15184904]

(26). Mancuso L, Jiirjens G, Hermane J, Harmrolfs K, Eichner S, Fohrer J, Collisi W, Sasse F, Kirschning A. Bioreduction of Aryl Azides during Mutasynthesis of New Ansamitocins. Org. Lett. 2013; 15:4442-4445. [PubMed: 23981134]

(27). Borodina I, Siebring J, Zhang J, Smith CP, van Keulen G, Dijkhuizen L, Nielsen J. Antibiotic overproduction in Streptomyces coelicolor A3(2) mediated by phosphofructokinase deletion. J. Biol. Chem. 2008; 283:25186-25199. [PubMed: 18606812]

(28). Wang L, Xie J, Deniz AA, Schultz PG. Unnatural Amino Acid Mutagenesis of Green Fluorescent Protein. J. Org. Chem. 2003; 68:174-176. [PubMed: 12515477]

(29). Wang F, Niu W, Guo J, Schultz PG. Unnatural Amino Acid Mutagenesis of Fluorescent Proteins. Ang. Chem. Int. Ed. 2012; 51:10132-10135.

(30). Reddington SC, Rizkallah PJ, Watson PD, Pearson R, Tippmann EM, Jones DD. Different Photochemical Events of a Genetically Encoded Phenyl Azide Define and Modulate GFP Fluorescence. Ang. Chem. Int. Ed. 2013; 52:5974-5977. 

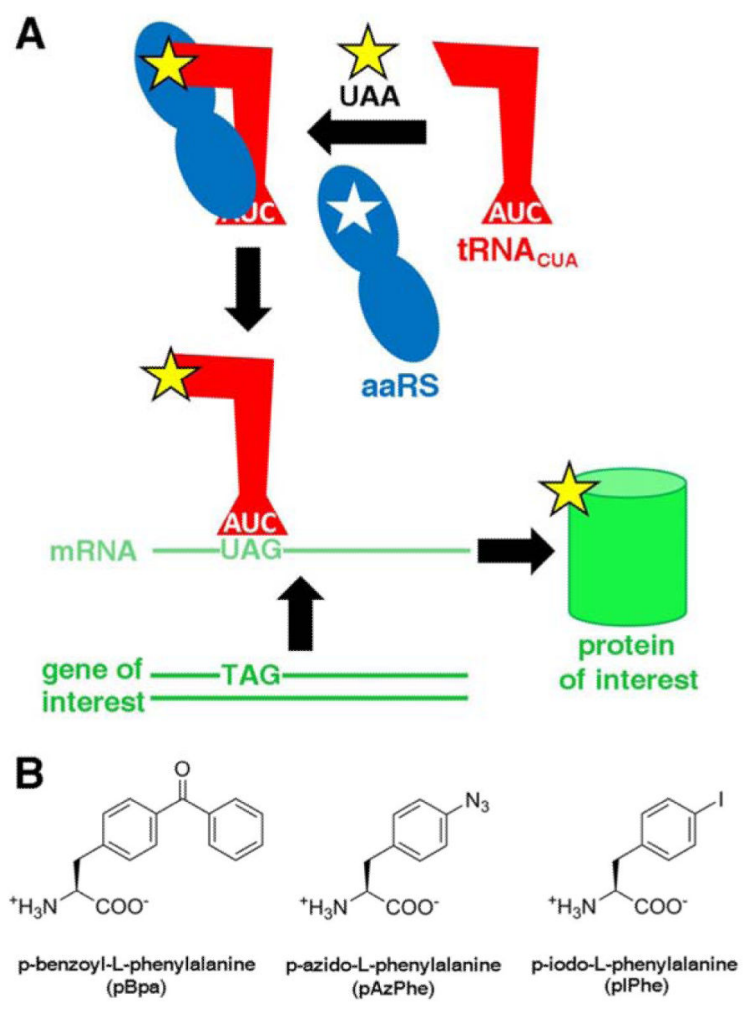

Figure 1.

a) Schematic overview of amber suppression-based unnatural amino acid (UAA) incorporation; b) structures of UAAs p-benzoyl-L-phenylalanine (pBpa), p-azido-Lphenylalanine (pAzPhe), and p-iodo L-phenylalanine (pIPhe) used in this study. 


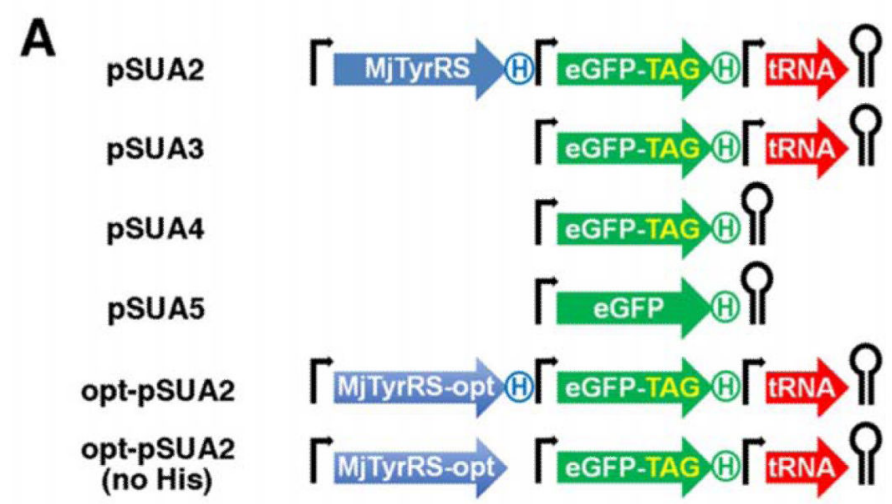

B

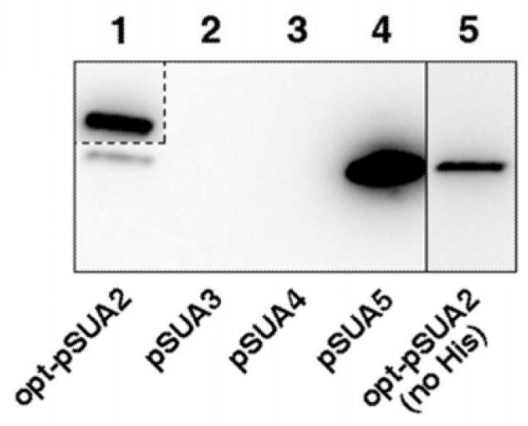

Figure 2.

a) Schematics of pSUA2, pSUA3, pSUA4, pSUA5, opt-pSUA2, and opt-pSUA2 (no His) vector inserts containing elements of the amber suppression reporter system; b) Anti-His Western blots of Ni-NTA affinity purified eGFP proteins obtained from $S$. venezuelae harboring opt-pSUA2 (lane 1), pSUA3 (lane 2), pSUA4 (lane 3), pSUA5 (lane 4), and optpSUA2 lacking the C-terminal His tag on MjTyrRS (lane 5). The molecular weights of MjTyrRS and eGFP are $35 \mathrm{kDa}$ and $27 \mathrm{kDa}$, respectively. 


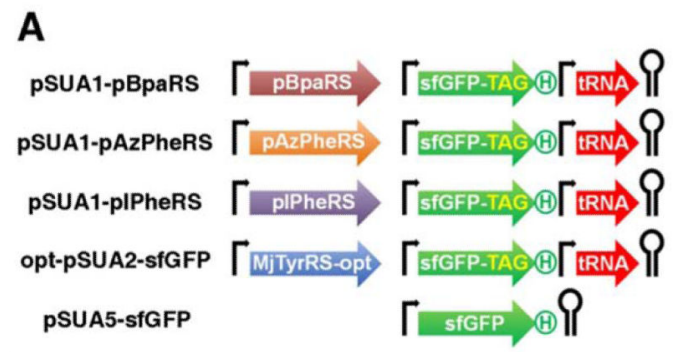

B

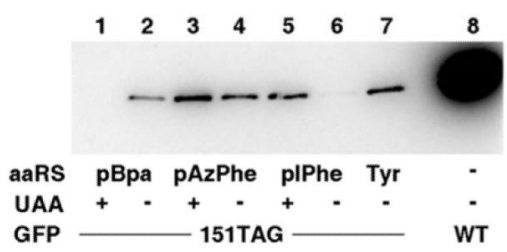

C

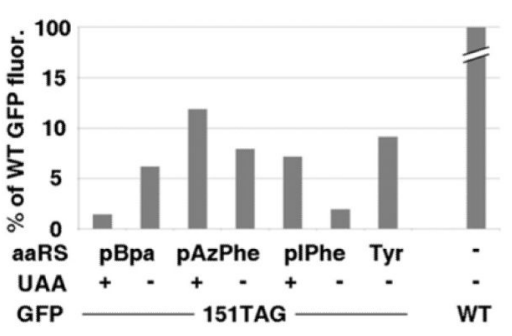

Figure 3.

a) Schematics of the pSUAl-pBpaRS, pSUAl-pAzPheRS, pSUAl-pIPheRS, opt-pSUA2sfGFP, and pSUA5-sfGFP vector inserts; b) Anti-His Western blot of Ni-NTA affinity pwified sfGFP proteins obtained from $S$. venezuelae harboring pSUAl-pBpaRS grown in the presence (lane 1) and the absence (lane 2) of $1 \mathrm{mM}$ pBpa, pSUAl-pAzPheRS grown in the presence (lane 3) and the absence (lane 4) of $\mathbf{1} \mathrm{mM}$ pAzPhe, pSUAl-pIPheRS grown in the presence (lane 5) and the absence (lane 6) of $1 \mathrm{mM}$ plPhe, opt-pSUA2-sfGFP (lane 7), and pSUA5-sfGFP (lane 8). The blot was imaged for $165 \mathrm{~s}$. c) Amber suppression efficiencies of the same eight protein samples used for Western blot analysis (Figure 3b), measured by fluorescence quantification, and normalized to wild-type sfGFP signal. GFP excitation was carried out at $485 \mathrm{~nm}$, bandwidth $9 \mathrm{~nm}$; and GFP emission was detected at 520 run, bandwidth 15 run. 


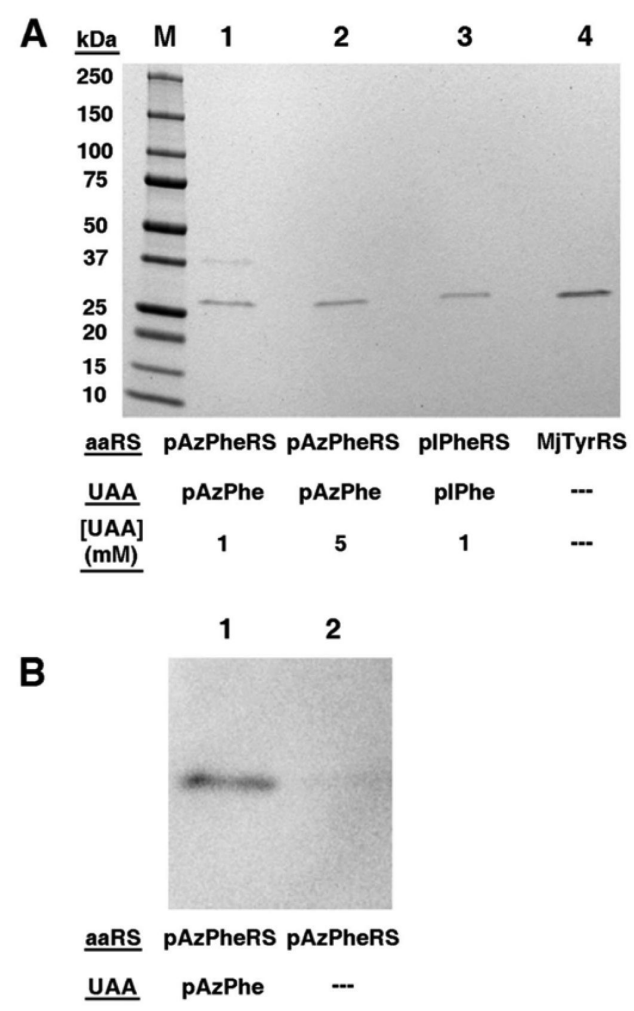

Figure 4.

a) SDS-PAGE of purified sfGFP proteins obtained from $S$. venezuelae harboring pSUAI pAzPheRS grown in the presence of I mM pAzPhe (lane I), pSUAl-pAzPheRS grown in the presence of $5 \mathrm{mM}$ pAzPhe (lane 2), pSUAI-plPheRS grown in the presence of I mM plPhe (lane 3), and opt-pSUA2-sfGFP (lane 4). b) Streptavidin Western blot of purified sfGFP proteins obtained from $S$. venezuelae harboring pSUAl-pAzPheRS grown in the presence (lane 1) and the absence (lane 2) of $1 \mathrm{mM}$ pAzPhe, incubated with $2 \mu \mathrm{M}$ DBCO-PEG $4_{4}$ biotin at $25^{\circ} \mathrm{C}$ for $12 \mathrm{~h}$, and re-purified by Ni-NTA affinity chromatography to remove excess DBCO-PEG4-biotin. The blot was imaged for $5 \mathrm{~s}$. 


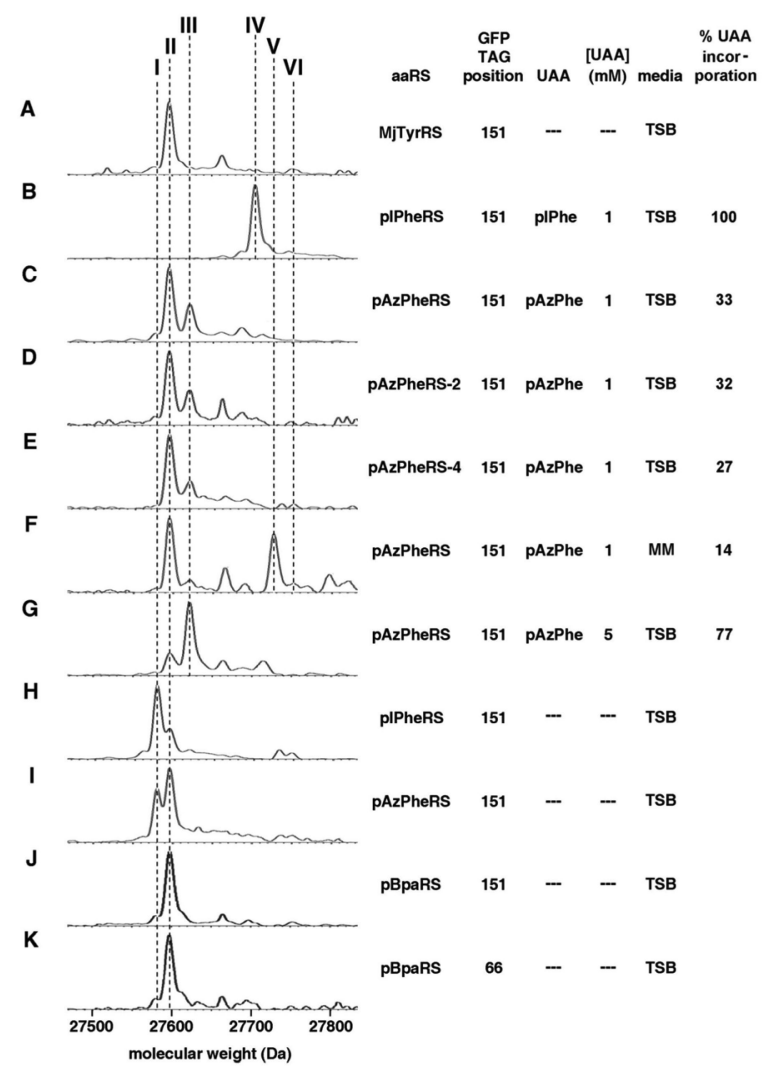

Figure 5.

Deconvoluted ESI-MS data of GFP proteins purified from $S$. venezuelae. The aaRS, GFP-

TAG, UAA, concentration of UAA, and growth media used for each protein expression experiment is shown to the right of each spectrum. The percentage of UAA incorporation, as estimated by the ratios of peak heights, is also shown to the right of each spectrum where appropriate. Peak I corresponds to GFP in which phenylalanine has been incorporated (Spectra H, I), Peaks II and V to GFP with tyrosine or $p$ amino---phenylalanine incorporated (Spectra A, C-K), Peak III and VI to GFP with pAzPhe incorporated (Spectra C-G), and Peak $N$ to GFP with pIPhe incorporated (Spectrum B). Peaks 1-N correspond to proteins with the N-terminal methionine removed, and Peaks V and VI to proteins with the Nterminal methionine retained (Spectrum F only). See Table S2 for observed and expected masses for each peak in each spectrum, and Figures S2-S12 for raw and deconvoluted ESIMS spectra. 

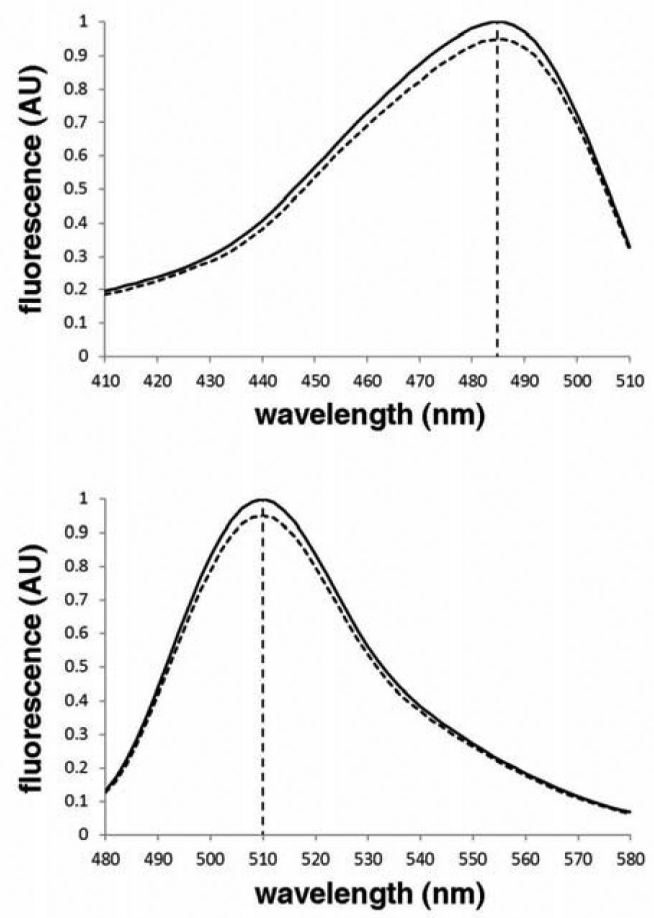

Figure 6.

Excitation (upper) and emission (lower) spectra of GFP proteins purified from $S$. venezue/ae harboring opt-pSUA2-sfUFP (solid line) and pSUA1-pBpaRS-Y66TAG (dashed line). The excitation spectrwn was obtained by excitation over the $410-510 \mathrm{~nm}$ range and detection of emission at $540 \mathrm{~nm}$; and the emission spectrum was obtained by excitation at $446 \mathrm{~nm}$ and detection of emission over the $480-580 \mathrm{~nm}$ range. To aid in comparison of peak shapes, both spectra were normalized, with the intensity of each dashed line spectrum adjusted to $95 \%$ of the intensity of the solid line spectrum at the excitation and emission maxima. 